\title{
A influência da anomia e do comprometimento organizacional sobre a intenção de rotatividade em organizações
}

\section{Influence of Anomie and Organizational Commitment on the Turnover Intention in Organizations}

\author{
Eliane Abreu ${ }^{1}$ \\ Marcos Aguiar de Souza ${ }^{2}$ \\ Luis Felipe de Oliveira Fleury ${ }^{3}$ \\ ${ }^{1}$ Universidade Federal Rural do Rio de Janeiro, Brasil \\ ${ }^{2,3}$ Universidade Federal do Rio de Janeiro, Brasil
}

Resumo. O contexto atual das organizações é de grande competição em um mercado cada vez mais marcado por mudanças. Para as empresas se manterem competitivas em tal cenário, devem investir em ações de recrutamento, seleção e treinamento e, principalmente, criar estratégias para reter seus talentos. No presente estudo pretendeu-se testar um modelo explicativo da intenção de rotatividade a partir dos preditores anomia organizacional e comprometimento organizacional. Participaram 325 trabalhadores de diferentes organizações. Os participantes responderam a instrumentos validados para mensurar as variáveis do estudo. As respostas foram submetidas a técnica de Modelagem de Equações Estruturais. Os resultados demonstraram que a anomia exerce influência negativa sobre o comprometimento e positiva sobre a intenção de sair da empresa.

Palavras-chave. Anomia organizacional, comprometimento organizacional, intenção de rotatividade, comprometimento calculativo, comprometimento normativo.

Abstract. The current context of organizations is of great competition in a market increasingly marked by change. For companies to remain competitive in such a scenario, they must invest in recruitment, selection, and training; as well as create strategies to retain their talents. The present study attempts to test an explanatory model of turnover intention with organizational anomia and organizational commitment as predictors. A total of 325 workers from different organizations participated in the study. Participants responded to validated instruments to measure study variables. The answers were subjected to Structural Equation Modeling. The results showed that anomie exerts a negative influence on commitment and a positive one on intention to leave the company.

Keywords. Organizational anomie, organizational commitment, turnover intention, calculative commitment. normative commitment.

${ }^{1}$ Eliane Abreu. Universidade Federal Rural do Rio de Janeiro, Brasil. Dirección Postal: Rua Anna Vasco, 18. Rio de Janeiro, Brasil. CEP: 23092620. E-mail: elianeabreu00@gmail.com

${ }^{2}$ Marcos Aguiar de Souza. Universidade Federal do Rio de Janeiro, Brasil. E-mail: maguiarsouza@uol.com.br

${ }^{3}$ Luis Felipe de Oliveira Fleury. Universidade Federal do Rio de Janeiro, Brasil. E-mail: luis_fleury@yahoo.com.br

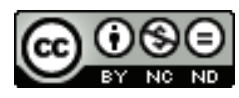

Esta obra está bajo una licencia de Creative Commons Reconocimiento-NoComercial-SinObraDerivada 4.0 Internacional. 


\section{Introdução}

A atualidade é marcada pela globalização que ampliou as fronteiras do mercado. As constantes alterações promovidas pelas tecnologias da informação estão redirecionando as formas de organização da produção para o aumento da produtividade e consequentemente maior lucro. Assim, há desafios diários para empresas que precisam reorganizar o modo de atuação dos trabalhadores (Bastos \& Costa, 2014).

É possível afirmar que os processos internos das organizações vêm sofrendo mudanças, dessa maneira, antigas práticas de gestão são revistas, como por exemplo: diminuição dos níveis hierárquicos, terceirização, descentralização e desvalorização das rígidas estruturas de cargos e tarefas (Malvezzi, 1995). Apesar da flexibilidade e da agilidade demonstrada por diversas organizações para o atendimento às demandas do mercado, em um aspecto as mesmas parecem não conseguir rápida adequação: o desenvolvimento de novas normas. Deste modo torna-se compreensível porque muitas vezes a organização funciona de modo diferente do que está prescrito no regulamento (Souza \& Ribas Júnior, 2013).

De acordo com Souza e Ribas Júnior (2013) a divergência existente entre as normas organizacionais e o cumprimento delas diz respeito à anomia organizacional. $\mathrm{O}$ termo anomia significa falta de normas ou não cumprimento dessas. De acordo com os autores, o mesmo surgiu na antiguidade, especificamente na Grécia, derivado de nomos (lei). Assim anomia é a negação do nomos, a inexistência de leis, desordem.

No século XIX, o sociólogo francês Émile Durkheim inicia a trajetória do termo anomia na Sociologia relacionando-o a fatos sociais negativos, como o suicídio e o aumento de condutas desviantes (Cohen, 1997). Posteriormente, Merton (1938) estudou as atitudes dos individuos quando em situação de anomia. De maneira geral, ambos os autores estavam preocupados na maneira como a ausência de normas no grupo social leva a alterações de comportamentos individuais que prejudicam o próprio grupo.

Segundo Durkheim (1893) existem três situações onde a anomia se manifesta mais claramente na sociedade, são elas: situação de crise econômica, de rápido crescimento econômico e em situações onde as instituições de Estado, responsáveis por regulamentar a sociedade, falham. Sob essa ótica, a anomia acontece pela diminuição na crença de que as instituições tem legitimidade para regulamentar a sociedade (Quintaneiro, Barbosa, \& Oliveira, 2002).

Merton (1938), por sua vez, apresenta uma nova concepção da teoria da anomia. De acordo com o autor a anomia é um colapso na estrutura cultural, em outras palavras, ocorre quando há uma incongruência entre as metas socialmente valorizadas e os meios legais disponíveis para o alcance de tais metas.

As sociedades, segundo Merton (1938) devem manter um equilibrio entre as metas sociais valorizadas e a disponibilização de meios legais para que todos os cidadãos tenham uma razoável chance de atingí-las. A percepção de que os caminhos normativos prescritos para alcance das metas não estão disponíveis de maneira igual, leva a uma situação de anomia social.

Após décadas de estudo, o conceito de anomia tem se expandido, sendo considerados tanto em níveis micro e macro (Zhao \& Cao, 2010). Assim, atualmente o termo anomia 
tem sido empregado em estudos de diversas generalidades, tais como as diferentes formas de atuação entre os países para justificar comportamentos eticamente suspeito (Cullen, Fisher, \& Applegate, 2000); a relação entre a pobreza, a mudança socio econômica, o homicídio e a anomia institucional (Kim, 2003); o crime de colarinho branco (Schoepfer \& Piquero, 2006); a relação entre a anomia e a saúde mental na América Latina (Quenza, 2009); a conduta desviante em jovens (Formiga, 2012); alguns estudos no contexto organizacional (Souza \& Ribas Júnior, 2013; Manrique de Lara \& Melián González, 2009).

Appelbaum, Iaconi e Matousek (2007) consideram que quando o comportamento do trabalhador diverge do que é prescrito pela norma as consequências afetam todos os níveis da organização causando impactos nos processos, na produtividade e retorno financeiro. Coccia (1998) define as normas organizacionais como um conjunto de atitudes, linguagens, fundamentos, premissas e regras que possibilitam a realização do trabalho em um ritmo ajustado. E de acordo com Applebaum et al. (2007), o espaço de trabalho é um ambiente onde há uma variedade de comportamentos e é necessário que esta diversidade de atitudes esteja em sintonia com as normas organizacionais para que a anomia não ocorra.

Manrique de Lara (2009) mostra que o contexto de anomia pode se apresentar quando há percepção do sentimento de indiferença partindo dos superiores, ou seja, o funcionário não se sente valorizado pelos gestores e/ou pela organização. Além disto, há também a imprevisibilidade nas relações de uma forma geral na organização que pode remeter ao não engajamento na empresa.

Essa imprevisibilidade é negativa, tendo em vista que o funcionário precisa sentir-se ajustado ao contexto da mesma, ou seja, o indivíduo precisa estar de acordo com os valores, a cultura e os objetivos organizacionais. Por ocasião desta realidade, caso isto não aconteça, os funcionários perdem a consciência moral, ou seja, já não sabem o que podem ou não fazer, perdem a autorregulação (Tsahuridu, 2006; 2011).

É válido ressaltar que a anomia organizacional pode interferir em outras variáveis como intenção de rotatividade e comprometimento organizacional. Em um contexto anômico o trabalhador se sente perdido, não sabe o que esperar dos gestores e colegas de trabalho (Souza \& Ribas Júnior, 2013). Assim, seu comprometimento tende a diminuir, podendo gerar intenção de sair da empresa (Bastos \& Costa, 2014). O comprometimento organizacional é entendido como um interesse do funcionário em se manter na empresa e desempenhar sua função de forma exemplar pelo sentimento de pertencimento à organização (Bhati \& Dixit, 2012).

O interesse pelo estudo do comprometimento organizacional está nos benefícios identificados tanto para o indivíduo como para a empresa (Allen \& Meyer, 1990; Jex, 2002; Jaros, 2007; Bastos \& Costa, 2014). No que diz respeito ao indivíduo, o comprometimento pode influenciar diversos comportamentos como absenteísmo, rotatividade e desempenho (Siqueira \& Gomide Júnior, 2014). Já para organização se busca descobrir o caminho para se desenvolver comportamentos que se relacionam com lealdade e a expressão "vestir a camisa da empresa" (Bastos \& Costa, 2014). 
De acordo com Bastos (1993), comprometimento organizacional é um tema sobre o qual não há muito consenso em relação a origem dos seus estudos. Becker (1960) pode ser apontado como referência do início da sistematização de conhecimento acerca desse fenômeno. É importante ressaltar que a expressão foi bastante empregada em estudos sociológicos da época, porém, pouco analisada em relação a variáveis organizacionais.

Mowday, Porter e Steers (1982) e Cappi e Araújo (2015) apresentam o comprometimento caracterizado por uma sólida convicção na receptividade de metas e valores organizacionais, vontade em se empenhar para empresa e grande desejo em continuar participando da organização. Chang (2001) por sua vez, sintetiza como o desejo de permanecer como membro da organização, o orgulho por pertencer a esta organização, a identificação com objetivos, metas e valores da empresa, o engajamento, o esforço, o empenho exercido em favor da instituição.

Para tanto, a vinculação de uma pessoa comprometida acontece de forma ativa (Mowday et al., 1982). Isto quer dizer, segundo Souza e Ribas Júnior (2013), que o comprometimento com algo demonstra um estado de bem-estar e supõe um empenho e aplicação para que o indivíduo realize as expectativas do que lhe foi solicitado.

O presente estudo tem como base o modelo de comprometimento organizacional de três componentes de Meyer e Allen (1991) que domina as pesquisas sobre comportamento organizacional (Jaros, 2007). Em tal modelo são considerados o comprometimento afetivo/atitudinal, de continuidade/normativo e o calculativo/instrumental.

O comprometimento afetivo ou atitudinal tem como base uma ligação emocional que o empregado desenvolve principalmente em função de experiências de trabalho positivas (Bastos \& Costa, 2014). Há assim uma identificação do indivíduo com as metas da empresa, com uma introjeção dos valores organizacionais que causam um sentimento de pertencimento. Tem sido considerado em diversos estudos como a forma de comprometimento mais efetiva (Dixit \& Bhati, 2012) e cresceu como o real possível vínculo organizacional (Mowday, Steers, \& Porter, 1979; Mowday et al., 1982).

De acordo com Melo et al. (2014), este tipo de comprometimento é uma espécie de apego, um envolvimento devido a identificação com a organização na interação entre indivíduo e organização. Os empregados permanecem na organização por que realmente desejam (Rego, 2003), por vontade própria.

O comprometimento normativo, por sua vez, é definido como um sentimento de obrigação moral em se manter na organização, onde o indivíduo assume internamente o reconhecimento de deveres morais para com a organização, e pensar em sair da mesma causa sentimentos de culpa (Wiener, 1982). Apesar do conceito relativamente simples, o comprometimento normativo é o menos conhecido (Jex, 2002; Bastos \& Costa, 2014), merecendo ainda investigações que se destinem a explicar como o indivíduo permanece fiel à organização mesmo em situações não tão favoráveis como, por exemplo, em crises financeiras, falta de recursos e atrasos no pagamento (Souza \& Ribas Júnior, 2013).

Finalmente, o comprometimento calculativo ou instrumental é definido como uma necessidade em se manter ou não na organização, a partir de outras possibilidades de 
emprego ou de falta delas (Rusbult \& Farrell, 1983). O indivíduo permanentemente compara a situação atual de trabalho com outras possibilidades e permanece na organização enquanto achar que a situação atual é a melhor que está a seu alcance.

Para Gibson, Ivancevich, Donnely e Konopaske (2006) funcionários comprometidos têm menor probabilidade de aceitar novos empregos ou pedir para sair da empresa, não gerando assim custos de rotatividade. Obter e manter funcionários talentosos na organização é, sem dúvida, uma preocupação constante das empresas (Lastres \& Albagli, 1999; Cohen, 2000).

O ambiente organizacional apresenta-se altamente competitivo e muito do diferencial entre as organizações está na capacidade e atuação dos trabalhadores. Desta forma as organizações têm se empenhado em rever suas estratégias de retenção de pessoal. $\mathrm{O}$ desejo de sair da empresa, chamado de intenção de rotatividade se refere à vontade que o trabalhador tem de se desligar da organização. Contudo, a perda de trabalhadores talentosos tem grande impacto em diversas frentes como equipe, gestão, produto ou serviço, custo, dentre outros (Lastres \& Albagli, 1999; Ferreira \& Freire, 2001).

Esses funcionários quando estão querendo sair da empresa não usam todo seu talento no desempenho de sua função, prejudicando assim o desempenho da organização (Milkovich \& Boudreau, 2000), enquanto nela permanecem. Logo, é de grande interesse entender o motivo que desperta essas pessoas para a saída da empresa.

Quanto mais qualificado o funcionário que sai da organização maior o investimento financeiro e mais tempo demora para que se possa substituí-lo (Simon \& Coltre, 2012). Ramlall (2003) conclui que o custo total da rotatividade perpassa por 150\% do salário referente ao cargo. Além do dispêndio financeiro há também a perda do conhecimento do funcionário que sai, ou o pouco investimento de conhecimento com o trabalhador que fica querendo sair. Portanto, para a organização se manter e crescer é fundamental preservar seus colaboradores (Spector, 2002).

De acordo com Chang (2001), rotatividade e intenção de rotatividade tem sido medidas separadamente. A intenção de rotatividade é considerada uma variável cognitiva final que pode levar à rotatividade. Assim, o autor ratifica a importância do estudo da intenção de rotatividade para compreender os determinantes da rotatividade na organização.

A rotatividade pode ser voluntária e involuntária. A primeira diz respeito ao desligamento do funcionário por vontade própria e parece ser a mais prejudicial à empresa, pois pode se tratar de trabalhadores com excelente desempenho e que atingem os interesses organizacionais. A segunda diz respeito não só aos desligamentos inevitáveis, tais como aposentadoria e os relacionados a doenças (Mobley, Griffeth, Hand, \& Meglino, 1979). Spector (2002) acrescenta que a demanda de mercado é um mediador de rotatividade, ou seja, poucas ofertas de trabalho diminui a perspectiva do trabalhador em pedir ou provocar sua saída da organização.

Considerando ainda os fatores preditores, Jha (2009) divide em três grandes grupos os motivos que elucidam a intenção de rotatividade. São eles: fatores individuais, organizacionais e mediadores. Os fatores individuais são relativos às características individuais como 
personalidade e habilidades. No que diz respeito aos fatores organizacionais, estes estão relacionados à remuneração, realização, reconhecimento, responsabilidade, possibilidade de crescimento profissional, condições de trabalho e segurança no trabalho, estresse, entre outros. E finalmente os fatores mediadores, ou seja, aqueles que não que podem ser influenciados por fatores individuais ou organizacionais e, consequentemente, exercerem influência sobre a intenção de rotatividade. Exemplos de fatores mediadores seriam comprometimento, satisfação no trabalho, autoestima, entre outros.

É importante considerar que nem toda rotatividade é negativa, tendo em vista que a mudança de alguns funcionários promove a renovação na organização. Siqueira e Pereira (1999) acrescentam que a perda de funcionários pouco produtivos é desejável. Assim, a renovação no quadro de funcionários é interessante para trazer novos talentos quando o trabalhador substituído já não atende mais às necessidades da empresa, quando já não apresenta comprometimento organizacional.

No que tange à intenção de rotatividade, Jex (2002) apresenta que de uma forma geral são vários os modelos preditivos adotados, e comprometimento organizacional é uma variável encontrada em quase todos eles. Entretanto, como é intuito considerar no presente estudo, a anomia organizacional tem sido negligenciada no que se refere a sua influência na intenção de rotatividade (Souza \& Ribas, 2013). Mais recentemente a literatura tem considerado preditores da intenção de rotatividade a percepção de suporte organizacional, políticas de gestão de pessoas e confiança organizacional (Oliveira, Gomide Júnior, Poli, \& Oliveira-Silva, 2018).

O objetivo do presente estudo foi testar um modelo explicativo da intenção de rotatividade utilizando os preditores de comprometimento organizacional e anomia organizacional. A hipótese analisada é que o comprometimento organizacional influencia negativamente a intenção de rotatividade. Bem como, também se hipotetiza a influência negativa da anomia organizacional na formação do comprometimento do trabalhador e positiva sobre a intenção de rotatividade.

\section{Método}

\section{Participantes}

Participaram do estudo 325 trabalhadores de diferentes empresas privadas do estado do Rio de Janeiro, sendo 132 homens e 193 mulheres, com idade variando de 20 a 62 anos (média de 28.49 anos, desvio padrão de 8.34 anos). O tempo de serviço variou de $1 \mathrm{a}$ 43 anos (média de 9.42 anos e desvio padrão de 9.02 anos). Os trabalhadores foram contactados por pesquisadores treinados para a coleta de dados em seus locais de trabalho com a autorização dos gestores.

Com o objetivo de verificar a significância da amostra para o referido estudo, calculou-se no pacote estatístico $G *$ Power 3.1, o qual, trata-se de um software destinado a cálculo do poder estatístico para a segurança amostral, testando a hipótese do ' $n$ ' ideal para a pesquisa a ser realizada. Neste programa, tem-se como base, não apenas o ' $n$ ' necessário para a pesquisa, mas, também, o tipo de cálculo a ser realizado (Faul, Erdfelder, Lang, \& Buchner, 2007). 
Para a coleta de dados deste estudo, considerando uma probabilidade de 95\% $(\phi<.05)$, magnitude do efeito amostral $(r \geq .50)$ e um padrão de poder hipotético $(\pi \geq .80)$, observou-se que a amostra especificada no estudo, revelou-se suficiente e significativa (tendo como indicadores: $t \geq 1.98 ; \pi \geq 1.00 ; p<.05$ ).

\section{Instrumentos}

Para mensuração da anomia organizacional foi utilizado o instrumento desenvolvido por Souza e Ribas Júnior (2013). Tal instrumento conta com 16 itens reunidos em um único fator, destinado a mensurar a percepção que o indivíduo tem do funcionamento das normas na organização em que trabalha. No estudo original, o instrumento obteve bons indicativos psicométricos $(\mathrm{KMO}=.90$; teste de esfericidade de Bartlett $=2620.96$; $p<.01 ; \alpha=.93)$.

A intenção de rotatividade foi mensurada com a utilização da escala de mesmo nome, validada para amostras brasileiras por Siqueira, Gomides Jr., Marques e Moura, (1997). A escala possui 3 itens reunidos em um único fator o instrumento obteve bons indicativos psicométricos $(\mathrm{KMO}=.84$; teste de esfericidade de Bartlett $=1522.96 ; p<.01 ; \alpha=.89)$.

O comprometimento organizacional foi mensurado com três instrumentos independentes. O comprometimento organizacional afetivo foi mensurado com a ECOA - Escala de Comprometimento Organizacional Afetivo, de autoria de Siqueira (1995). O instrumento é composto por 18 itens que formam um único fator. Foi obtido no estudo original um índice de precisão (alfa de Cronbach) de .95.

O comprometimento organizacional calculativo foi mensurado com utilização da ECOC (Escala de Comprometimento Organizacional Calculativo). Trata-se de uma escala multidimensional, composta por 15 itens divididos em 4 fatores (perdas sociais no trabalho, perdas de investimentos feitos na organização, perdas de retribuições organizacionais e perdas profissionais). O instrumento também foi desenvolvido por Siqueira (1995). O índice de confiabilidade (alfa de Cronbach) foi de .92 .

Finalmente, o comprometimento organizacional normativo foi mensurado com utilização da ECON (Escala de Comprometimento Organizacional Normativo), desenvolvida por Siqueira (1995). A escala é composta por 7 itens reunidos em um único fator, apresentando índice de precisão (alfa de Cronbach) de .86.

\section{Procedimiento}

Todos os procedimentos adotados nesta pesquisa seguiram as orientações previstas na Resolução 196/96 do Conselho Nacional de Saúde (1996) e da Resolução 016/2000 do Conselho Federal de Psicologia (2000). Colaboradores com experiência prévia na administração de instrumentos em Psicologia foram responsáveis pela coleta dos dados, e apresentaram-se nas empresas como interessados em conhecer as opiniões e os comportamentos dos colaboradores sobre as situações descritas no instrumento.

Solicitou-se a colaboração voluntária das pessoas no sentido de responderem um breve questionário. Após ficarem cientes das condições de participação na pesquisa, assinaram um termo de Consentimento Livre e Esclarecido. Foi-lhes dito que não havia resposta 
certa ou errada. A todos foi assegurado o anonimato das suas respostas informando que estas seriam tratadas em seu conjunto.

Análise de dados

Foi utlizado o software estatístico SPSS v. 20 e o software para Modelagem de Equações Estruturais AMOS v. 18. Dentre os principais parâmetros considerados na análise de equações estruturais na análise da adequação do modelo proposto, merecem destaque o $\chi^{2}$ (qui-quadrado), o Root Mean Square Residual (RMR), Goodness-of-Fit Index (GFI), o Comparative Fit Index (CFI) e o Root-Mean-Square Error of Approximation (RMSEA) (Byrne, 1989; Hair; Anderson; Tatham \& Black, 2005; Maroco, 2010).

$\mathrm{O} \chi^{2}$ (qui-quadrado) tem como objetivo testar a probabilidade do modelo teórico se ajustar aos dados, quanto maior este valor pior o ajustamento. Este tem sido pouco empregado em pesquisas, sendo mais comum considerar sua razão em relação aos graus de liberdade $\left(\chi^{2} / \mathrm{gl}\right)$. Neste caso, valores até 5 indicam um ajustamento adequado.

O RMR é utilizado para avaliar o ajustamento do modelo teórico aos dados, na medida em que a diferença entre os dois se aproxima de zero. Para um modelo bem ajustado, o valor ideal é menor que 05 .

O GFI e o AGFI são correspondentes ao $\mathrm{R}^{2}$ em regressão múltipla. Portanto, indicam a proporção de variância e covariância nos dados explicada pelo modelo. Estes variam de $0 \mathrm{a}$ 1 , sendo que valores na casa dos .80 e .90 , ou acima, indicam um ajustamento satisfatório.

O Comparative Fit Index (CFI) compara o modelo estimado e o modelo nulo, considerando valores mais próximos de 1 como indicadores de ajustamento satisfatório. $\mathrm{O}$ indicativo RMSEA é considerado um indicador de pobreza de ajuste. Assim, valores altos indicam um modelo não ajustado. Tem sido considerado como ideal que o RMSEA se situe entre .05 e .08. Porém, como destaca Garson (1998), valores até 10 podem ser aceitos.

\section{Resultados}

Inicialmente foi realizada uma análise descritiva dos dados, visando identificar possíveis correções a serem feitas na planilha, de modo a tornar mais fidedigna à análise pretendida. Posteriormente, apesar de todos os instrumentos utilizados no estudo estarem validados para amostras brasileiras, realizou-se uma Análise Fatorial Confirmatória dos mesmos, de modo a atestar a confiabilidade de cada instrumento na amostra do estudo.

Os procedimentos de Análise Fatorial Confirmatória permitiram constatar a adequação dos instrumentos para fins da investigação proposta. Foram então compostos os fatores de cada escala, utilizando-se a média dos escores obtidos pelos participantes do estudo. Na tabela 1 são apresentados os dados iniciais, com base na estatística descritiva (média, mediana e desvio padrão), cabe destacar que todos os instrumentos variam em uma pontuação de $1 \mathrm{a}$ 5 pontos, quanto mais próximo de 5 maior a presença do fenômeno na amostra.

Em seguida foi testado o modelo de influência da anomia organizacional e do comprometimento organizacional sobre a intenção de rotatividade. Onde a hipótese é de que o comprometimento exerce influência negativa significativa sobre a intenção de 
Tabela 1

Média, Mediana e Desvio Padrão da amostra total nos instrumentos do estudo

\begin{tabular}{lccc}
\hline Variável & Média & Mediana & Desvio Padrão \\
\hline Anomia Organizacional & 2.65 & 2.59 & .72 \\
Comprometimento Organizacional Afetivo & 3.19 & 3.23 & .81 \\
Comprometimento Organizacional Calculativo & 2.37 & 2.33 & .74 \\
Comprometimento Organizacional Normativo & 2.26 & 2.20 & .80 \\
Intenção de Rotatividade & 2.82 & 2.67 & 1.14 \\
\hline
\end{tabular}

rotatividade. Em relação à anomia a hipótese é que a anomia exerce influência positiva significativa sobre a intenção de rotatividade (Veja a figura 1).

$\mathrm{Na}$ tabela 2 é possível observar as estimativas de influência das variáveis sobre a intenção de rotativdade. Nota-se que apenas o Comprometimento Organizacional Normativo não exerceu influência significativa sobre a intenção de deixar a organização.

Pelos dados apresentados, foi possível testar o modelo do estudo com o uso da Modelagem por Equações Estruturais. De acordo com a tabela 3, a anomia organizacional influenciou positivamente a intenção de rotatividade. Desse modo, os resultados mostram que a percepção de ausência de normas no grupo desperta ou potencializa a vontade do indivíduo em deixar a empresa. Provavelmente, um dos motivos que justificam esta influência é o fato de o funcionário se sentir perdido, sem direcionamento em função do clima de desorientação e incertezas.

O comprometimento organizacional afetivo e calculativo, por sua vez, influenciaram negativamente a intenção de rotatividade. Isto quer dizer, no que diz respeito ao aspecto afetivo, que há pouca probabilidade do indivíduo que está na empresa por sentir à vontade, por gostar, com sentimento de pertencimento, desejar sair. No mesmo sentido, o comprometimento calculativo demonstra que o funcionário avalia durante a maior parte do tempo os prós e contras de estar nela, e conclui positivamente em favor da organização. Dito de outra forma, o trabalhador que constata perder vantagens sem possibilidades de reposição destas ou de investimentos feito até momento pela organização não demosntra intenção de deixar a empresa.

Já o comprometimento organizacional normativo não influenciou a intenção de rotatividade. É importante considerar que o comprometimento normativo contou com a menor média. Este resultado indica que o comprometimento normativo apresentado pelos participantes foi insuficiente para influenciar a intenção de rotatividade. De fato, o mercado de trabalho atualmente não tem uma visão tão negativa do trabalhador que muda de emprego como se considerava décadas atrás. É visto com naturalidade o fato do trabalhador procurar melhores condições de trabalho, melhores salários e um emprego que ofereça o nível de desafio que lhe é satisfatório.

Em seguida testou-se o modelo de impacto da anomia organizacional sobre o comprometimento organizacional. Onde a hipótese é de que a anomia organizacional 


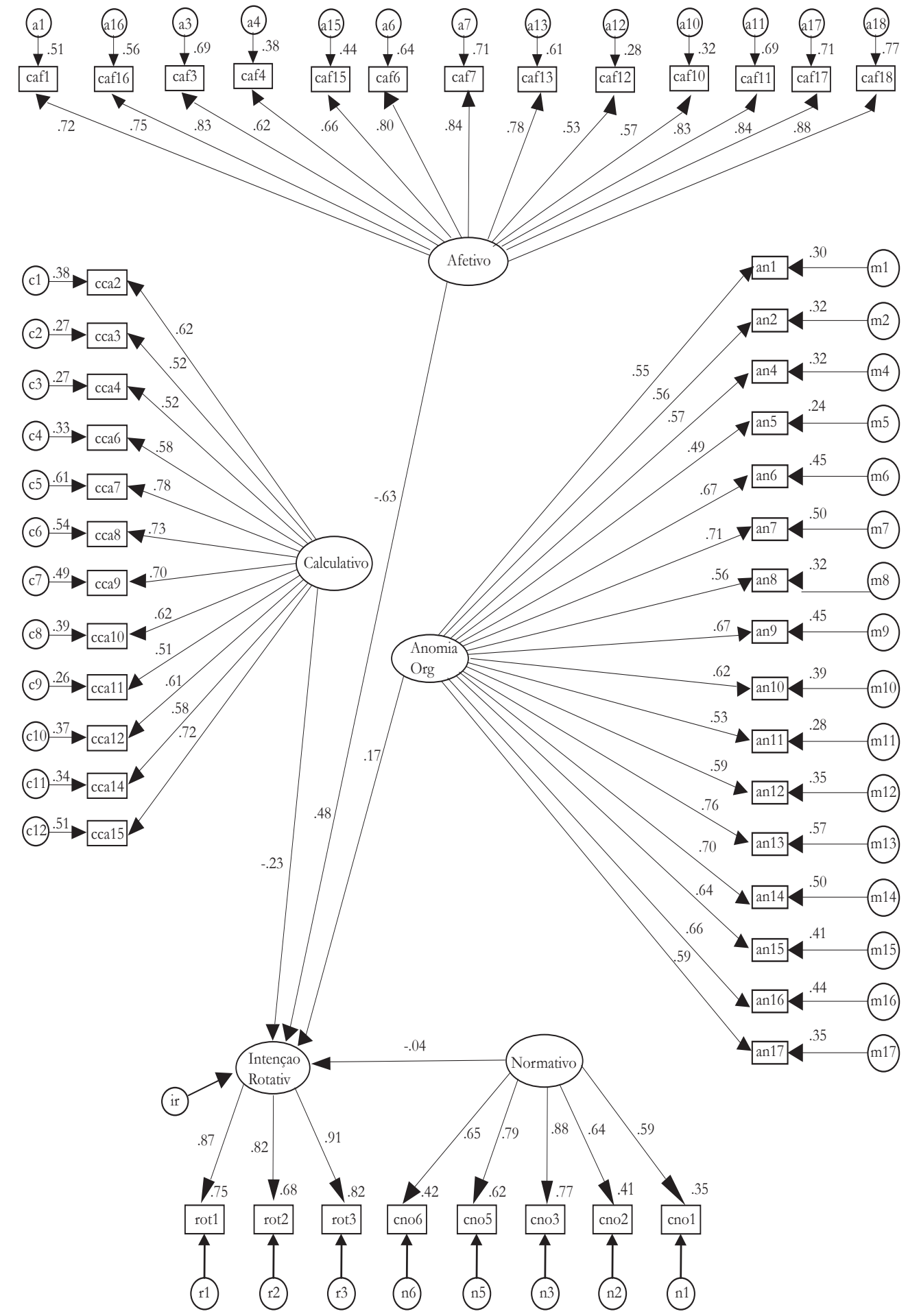

Figura 1. Teste do modelo do estudo com o uso da Modelagem por Equações Estruturais 
Tabela 2

Influência da anomia e do comprometimento organizacional sobre a intenção de rotatividade

\begin{tabular}{lcc}
\hline \multicolumn{1}{c}{ Variáveis independentes } & Variável dependente & Estimativa \\
\hline Anomia Organizacional & & $.17^{* *}$ \\
Comprometimento Organizacional Afetivo & Intenção de Rotatividade & $-.63^{* *}$ \\
Comprometimento Organizacional Calculativo & & $-.23^{* *}$ \\
Comprometimento Organizacional Normativo & & -.04 n.s. \\
\hline
\end{tabular}

$* * \mathrm{p}<.01$. n.s. - não significativo

Tabela 3

Parâmetros de ajuste do modelo de influência da anomia e do comprometimento organizacional sobre a intenção e rotatividade

\begin{tabular}{lcc}
\hline \multicolumn{1}{c}{ Parâmetros } & Valor obtido & Valor ideal \\
\hline$\chi^{2}$ & 131.00 & - \\
$\chi^{2} / g l$ & 2.67 & Até 5 \\
RMR & .04 & Menor que .05 \\
GFI & .94 & Acima de .80 \\
AGFI & .91 & Acima de .80 \\
CFI & .95 & Próximo a 1 \\
RMSEA & $.07(.05-.08)$ & Menor que .10 \\
\hline
\end{tabular}

influencia de forma negativa e significativa o comprometimento organizacional. Tal modelo tem base nos efeitos nocivos exercidos pela anomia organizacional que são apontados na literatura (Souza \& Ribas, 2013; Bastos \& Costa, 2014). Todo pode ser observado na figura 2 e nas tabelas 4 e 5 .

Observa-se nos resultados que a anomia organizacional influenciou negativamente o comprometimento afetivo e normativo, de forma significativa $(\phi<.01)$. Esta influência negativa remete ao quadro de que quanto maior o estado de anomia, menor o comprometimento com a empresa. É importante considerar que o comprometimento afetivo foi o que maior influência teve da anomia organizacional. Tal resultado indica que o sentimento positivo que é nutrido pelo trabalhador em relação à organização a que pertence é mais facilmente abalado em uma situação na qual existe uma incerteza no dia a dia de trabalho sobre as normas que regem a organização.

A influência da anomia organizacional não foi significativa apenas no que se refere ao comprometimento calculativo. A justificativa para tal resultado é que o comprometimento calculativo depende não somente do contexto vivenciado na organização, como no caso do comprometimento afetivo e do comprometimento normativo.

Também é importante a avaliação de possíveis alternativas de trabalho disponibilizadas pelo mercado. Especificamente o trabalhador avalia se teria melhores condições indo para 


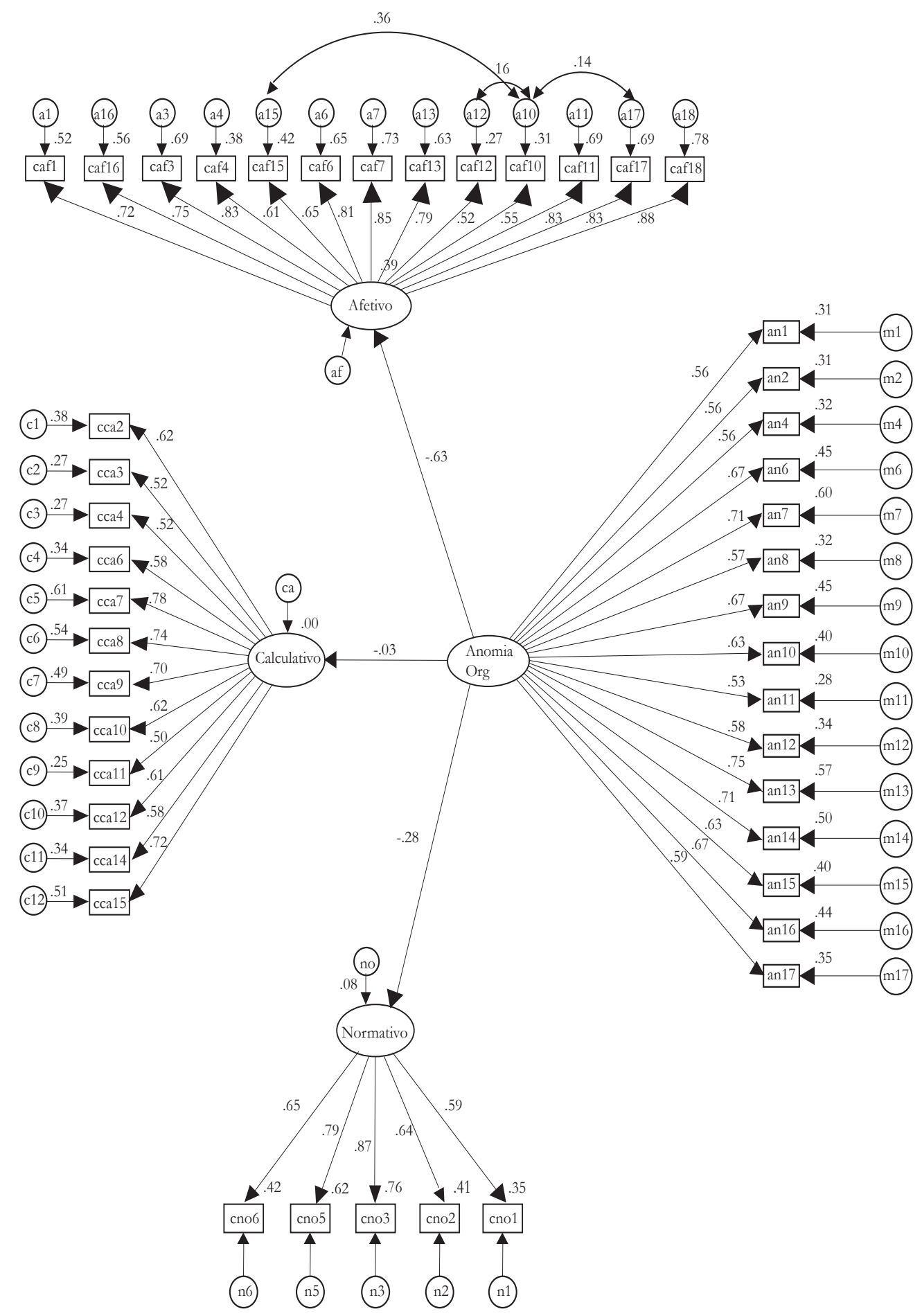

Figura 2. Modelo explicativo do impacto da anomia organizacional sobre o comprometimento organizacional 
Tabela 4

Impacto da Anomia Organizacional sobre o Comprometimento Organizacional

\begin{tabular}{cll}
\hline Variável Independente & Variáveis dependentes & Estimativa \\
\hline Anomia Organizacional & Comprometimento Organizacional Afetivo & $-.63^{* *}$ \\
& Comprometimento Organizacional Calculativo & -.03 n.s. \\
& Comprometimento Organizacional Normativo & $-.28^{* *}$ \\
\hline
\end{tabular}

** $\mathrm{p}<.01$. n.s. - Não significativo

Tabela 5

Parâmetros do modelo de impacto da Anomia Organizacional sobre o Comprometimento Organizacional

\begin{tabular}{|c|c|c|}
\hline Parâmetros & Valor obtido & Valor ideal \\
\hline$\chi^{2}$ & 177.00 & - \\
\hline$\chi^{2} / g 1$ & 1.91 & Até 5 \\
\hline RMR & .04 & Menor que .05 \\
\hline GFI & .84 & Acima de .80 \\
\hline AGFI & .85 & Acima de .80 \\
\hline CFI & .89 & Próximo a 1 \\
\hline RMSEA & $.05(.05-.07)$ & Menor que .10 \\
\hline
\end{tabular}

um outro emprego e mesmo se essa alternativa é uma realidade, mesmo em um mercado caracterizado pela escassez de oferta de empregos, como é atualmente o caso na cidade do Rio de Janeiro.

\section{Discussão}

O presente estudo teve como objetivo geral testar um modelo explicativo da intenção de rotatividade a partir dos preditores anomia organizacional e comprometimento organizacional (afetivo, normativo e calculativo). Cabe ressaltar que a hipótese do presente estudo de influência negativa do comprometimento organizacional, sobretudo em sua vertente afetiva, sobre a intenção de rotatividade foi confirmada.

E também a influência negativa da anomia sobre o comprometimento bem como, sua influência positiva sobre a intenção de sair da empresa. Tais achados estão de acordo com a literatura consultada sobre o tema do comprometimento organizacional e da intenção de rotatividade (Yao, Qiu, \& Wei, 2018; Miedaner, Kuntz, Enke, Roth, \& Nietzsche, 2018; Van der Heijden, Peeters, Le Blanc, \& Van Breukelen, 2018).

A anomia organizacional e o comprometimento organizacional apresentaram-se como importantes variáveis na explicação da intenção de rotatividade nas organizações, de maneira que a presente investigação está em consonância com outros estudos que vêm sendo realizado sobre a temática da intenção de rotatividade (Bastos \& Costa, 2014; Oliveira, Gomide Júnior, Poli, \& Oliveira-Silva, 2018). 
Quanto ao trabalhador, o comprometimento organizacional afetivo e calculativo, além do tempo de serviço e idade, são alguns dos fatores responsáveis pela manutenção do mesmo na empresa. No que diz respeito ao comprometimento, o afetivo destaca-se entre os demais quando o assunto é relacionado a anomia e intenção de rotatividade. Dessa maneira, os achados nesta pesquisa corroboram o exposto em estudos pregressos sobre a importância de trabalhadores afetivamente comoprometidos com o trabalho (Bastos et al., 2014; Bhati \& Dixit, 2012; Simon \& Coltre, 2012; Jex, 2002).

O bom funcionamento das normas é primordial para manter seus funcionários em uma organização. Isto quer dizer que estabelecer e divulgar regras claras traz como consequência não só diminuição na intenção de rotatividade como também proporciona um aumento do comprometimento, principalmente no que diz respeito ao comprometimento afetivo, relacionado ao desejo em se manter na empresa por vontade própria. O motivo para tal realidade está pautado no fato de que só com normas claras os funcionários sabem como proceder, o que esperar do colega de trabalho e da empresa. Assim, a presente pesquisa está de acordo com a teoria (Manrique de Lara \& Melián González, 2009; Manrique de Lara, 2009; Zhao \& Cao, 2010; Formiga, 2012) ao apontar que em um ambiente organizacional com regras, os trabalhadores se sentem mais seguros e não apresentam interesse em sair da empresa, bem como desenvolvem melhor seu trabalho.

O estudo contribui para demonstrar um fator que tem sido negligenciado por parte de pesquisadores e gestores na área organizacional: as consequências para o trabalhador de um ambiente em que as regras não são claras e/ou não funcionam. Sugere-se assim uma atenção maior por parte de tais profissionais sobre como está o funcionamento das normas organizacionais. Ou seja, faz-se necessário observar a distância que há entre a forma como a organização foi prevista para funcionar e como ela realmente funciona e o grau de incerteza que caracteriza a relação do trabalhador com a organização.

O baixo resultado obtido no presente estudo no que se refere ao comprometimento normativo parece ser um outro indicativo que merece atenção. É possível que, dadas as características atuais do mercado de trabalho, tal comprometimento venha a ser ainda mais diminuído ou mesmo extinto. Assim, relações mais claras de trabalho, que favoreçam o vínculo do trabalhador com a organização devem ser estimuladas pelos setores responsáveis pela gestão de pessoas.

Finalmente, sugere-se que novas investigações sejam realizadas, diante da recente mudança das leis trabalhistas no Brasil. É possível que o momento de transição diante da mudança das regras que regem a relação trabalhador/empregador gere uma situação de anomia, ainda que não esteja claro para a maioria dos trabalhadores como avaliar o conjunto de mudanças e como tais mudanças terão impacto no dia a dia de trabalho.

\section{Referências}

Allen, N. J. \& Meyer, J. P. (1990). The measurement and antecedents of affective, continuance and normative commitment to the organization. Journal of Occupational Psychology, 63(1), 1-18. doi: 10.1111/j.2044-8325.1990. tb00506.x 
Appelbaum, S. H., Laconi G. D., \& Matousek A. (2007). Positive and negative deviant workplace behaviors: causes, impacts, and solutions. Corporate Governance: The international journal of business in society, 7(5), 586-598. doi: 10.1108/14720700710827176

Bastos, A. V. B. (1993). Comprometimento organizacional: um balanço dos resultados e desafios que cercam essa tradição de pesquisa. Revista de Administração de Empresas, 33(3), 52-64. doi: 10.1590/S0034-75901993000300005

Bastos, A. V. B., \& Costa, F. M. (2014). Comprometimento Organizacional: Bases para uma Abordagem Processual. Psicologia: Teoria e Pesquisa, 30(3), 329-337. doi: 10.1590/S0102-37722014000300010

Bastos, A. V. B., Maia, L. G. M., Rodrigues, A. C. D. A., Macambira, M. O., \& BorgesAndrade, J. E. (2014). Vínculos dos indivíduos com a organização: análise da produção científica brasileira 2000-2010. Psicologia: Teoria e Pesquisa, 30(2), 153-162.

Becker, H. (1960). Notes on the concept of commitment. The American Journal of Sociology, 66(1), 32-40.

Bhati, M., \& Dixit, V. (2012). A Study about Employee Commitment and its impact on Sustained Productivity in Indian Auto-Component Industry. European Journal of Business and Social Sciences, 1(6), 34-51.

Byrne, B. M. (1989). A primer of LISREL: Basic applications and programming for confirmatory factor analytic models. New York: Springer-Verlag.

Cappi, M. \& Araujo, B. F. V. B. (2015). Satisfação no trabalho, comprometimento organizacional e intenção de sair: um estudo entre as gerações X e Y. Read Revista. Eletrônica de Administração. 82(3), 576-600. doi: 10.1590/14132311.0782014.54843

Chang, J. Jr. (2001). Gestão de Pessoas Através do Desenvolvimento do Comprometimento Organizacional: uma abordagem holística e simultânea dos determinantes envolvidos nesse processo. São Paulo: FEA/USP Tese de Doutorado.

Coccia, C. (1998). Avoiding a "toxic” organization. Nursing Management, 29(5), 32-34.

Cohen, A. K. (1997). An elaboration of anomie theory. Nikos Passas \& Robert Agnew (Eds.). The future of anomie theory. Boston: Northeastern University Press.

Cohen, A. K. (2000). The relationship between commitment forms and work outcomes: A comparison of the three models. Human Relations, 53(3), 387-417. doi: $10.1177 / 0018726700533005$

Cullen, F. T., Fisher, B. S., \& Applegate, B. K. (2000). Public opinion about punishment and corrections. In M. Tonry (Ed.), Crime and justice: A review of research. Chicago: University of Chicago Press.

Dixit, V. \& Bhati, M. (2012). A study About Employee Commitment and its Impact on Sustained Productivity in India Auto-Component Industry European. Journal of Business and Social Sceinces. 1(6), 44-45. 
Durkheim, E. (1893). De la division du travail social. Paris: Alcan.

Faul, F., Erdfelder, E., Lang, A. G., \& Buchner, A. (2007). G*Power 3: A flexible statistical power analysis program for the social, behavioral, and biomedical sciences. Behavior Research Methods, 39, 175- 191. doi: 10.3758/BF03193146

Ferreira, M. C., \& Freire, O. N. (2001). Carga de Trabalho e Rotatividade na Função de Frentista. Revista de Administração Contemporânea, 5(2), 175-200. doi: 10.1590/ S1415-65552001000200009

Formiga, N. S. (2012). Anomia social, sentimento anômico e condutas desviantes: verificação de um modelo teórico em jovens. Revista Latinoamericana de Psicología Social Ignacio Martín-Baró, 1, 56-79.

Garson, G. D. (1998). Introduction. Social Science Computer Review, 16(1), 3-3. doi:10.1177/089443939801600101

Gibson, J. L., Ivancevich, J. M., Donnely, Jr. J. H., \& Konopaske, R. K. (2006). Organizações: comportamento, estrutura e processos. São Paulo: McGraw-hill.

Hair, J. F., Anderson, R. E., Tatham, R. L., \& Black, W. (2005). Análise Multivariada de Dados. Porto Alegre: Bookman.

Jaros, S. (2007). Meyer and Allen Model of Organizational Commitment: Measurement Issues. Journal of Organizational Behavior, 6(4), 7-25.

Jex, S. M. (2002). Organizational psychology: A scientist practitioner approach. New York: John Wiley \& Sons.

Jha, S. (2009). Determinants of employee turnover intentions: A review. Management Today, 9(2), 26-33.

Kim, S. W. (2003). Anomie, institutions, and crime: The role of social institutions in the relationship between socioeconomic change and crime in Russia. University of Oklahoma, Department of Sociology. Chair of PhD Dissertation.

Lastres, H. M. M., \& Albagli, S. (1999). Informação e globalização na era do conbecimento. Rio de Janeiro: Campus.

Malvezzi, S. (1995). A Carreira Profissional ainda Depende de Ascensão na Hierarquia do Poder? Marketing Industrial, 1(1), 30-40.

Manrique de Lara, P. Z. (2009). La Anomia como Moderador de la Relación entre Percepciones de Justicia Organizativa y el Uso Negligente de Internet en el Trabajo. Revista de Psicología del Trabajo y de las Organizaciones, 25(2), 99-112.

Manrique de Lara, P. Z. \& Melián-González, S. (2009). The role of anomia on the relationship between organizational justice perceptions and organizational citizenship online behaviors. Journal of Information, Communication and Ethics in Society, 7(1), 72-85. doi: .1108/14779960910938106

Maroco, J. (2010). Análise de Equações Estruturais: fundamentos teóricos, software \& aplicações. Portugal: Report Number. 
Melo, M. A. S., Bezerra J. C. B., Coleta, M. F. D., Puente-Palacios, K. E., Puente-Palacios, J. A. D., \& Bezerra, A. L. Q. B. (2014). Comprometimento Organizacional de trabalhadores da Vigilância Sanitária em municípios do estado de Goiás. Trabalho, Educação e Saúde, 12(3), 655-677. doi: 10.1590/1981-7746-sip00018.

Merton, R. K. (1964). Anomie, Anomia and Social Interaction: Contexts of Deviant Behavior, in: Marshall Clinard (Hrsg.), Anomie and Deviant Behavior. New York: The Free Press, 213-242.

Merton, R. K. (1938). Social Structure and Anomie. American Sociological Review, 3, 672682. doi: $10.2307 / 2084686$

Meyer, J. P., \& Allen, N. J. (1991). A three-component conceptualization of organizational commitment. Human Resource Management Review, 1(1), 61-89. doi: 10.1016/10534822(91)90011-Z

Miedaner, F., Kuntz, L., Enke, C., Roth, B., \& Nietzsche, A. (2018). Exploring the differential impact of individual and organizational factors on organizational commitment of physicians and nurses. BMC Health Services Research, 18(1), 180. doi: 10.1186/s12913-018-2977-1

Milkovich, G. T., \& Boudreau, J. W. (2000). Administração de Recursos Humanos. São Paulo: Atlas.

Mowday, R. T. \& Steers, R. M. \& Porter, L. W. (1979). The Measurement of Organizational Commitment. Journal of Vocational Behavior, 14(2), 224-247. doi: 10.1016/00018791(79)90072-1

Mowday, R. T., Porter, L. W., \& Steers, R. M. (1982). Employee-organization linkages: the psychology of commitment, absenteeism, and turnover. New York: Academic Press.

Mobley, W. H., Griffeth, R. W., Hand, H. H., \& Meglino, B. N. (1979). Review and conceptual analysis of the employee turnover process. Psychological Bulletin, 86(3), 493-522. doi: 10.1037/0033-2909.86.3.493

Oliveira, A. F., Gomide Júnior, S., Poli, B. V. S., \& Oliveira-Silva, L. C. (2018). Análise dos Fatores Organizacionais Determinantes da Intenção de Rotatividade. Trends Psychology (Online), 26(2), 1031-1042. doi: 10.9788/tp2018.2-18pt.

Quenza, C. J. P. (2009). National Identity, Anomie and Mental Health in Latin America. Current Sociology, 57(6), 851-870. doi: 10.1177/0011392109342222

Quintaneiro, T., Barbosa, M. L. O., \& Oliveira, M. G. (2002). Um toque de clássicos: Marx, Dürkheim e Weber. $2^{\mathrm{a}}$ ed., Belo Horizonte: Editora UFMG.

Ramlall, S. (2003). Managing Employee Retention as a Strategy for Increasing Organizational Competitiveness. Applied H.R.M. Research, 8(2), 63-72.

Rego, A. (2003). Comprometimento Organizacional e Ausência Psicológica - afinal quantas dimensões? Revista de Administração de Empresas, 43(4), 25-35. doi: 10.1590/S0034-75902003000400003 
Rusbult, C. E., \& Farrell, D. (1983). A longitudinal test of investment model: The impact on job satisfaction, job commitment, and turnover of variations in rewards, costs, alternatives, and investments. Journal of Applied Psychology, 68(3), 429-438. doi: 10.1037/0021-9010.68.3.429

Schoepfer, A., \& Piquero, N. L. (2006). Exploring white-collar crime and the American dream: A partial test of institutional anomie theory. Jornal de Justiça Criminal. 34(3), 227-235.

Simon, J., \& Coltre, S. M. (2012). O comprometimento organizacional afetivo, instrumental e normativo: estudo de caso de uma empresa familiar. Qualitas Revista Eletrônica, 13(1), 1-20. doi: 10.18391/qualitas.v13i1.1338

Siqueira, M. M. M. (1995). Antecedentes de comportamento de cidadania organizacional: a análise de um modelo pós-cognitivo. (Tese de Doutorado). Instituto de Psicologia da Universidade de Brasília.

Siqueira, M. M. M. (2001). Comprometimento organizacional afetivo, calculativo e normativo: evidencias acerca da validade discriminante de três medidas brasileiras. [Trabalho completo]. In Associação Nacional de Pós-Graduação em Administração (Org.), XXV EnANPAD. Anais do Em ANPAD 2001 (CD-ROM). Campinas: Autor.

Siqueira, M. M. M., \& Gomide Jr., S. (2014). Vínculos do indivíduo com o trabalho e com a organização. In: J. C. Zanelli, J. E. Borges-Andrade, A. V. B. Bastos (orgs.). Psicologia, organizacões e trabalho no Brasil. Porto Alegre: Artmed.

Siqueira, M. M. M. \& Pereira, S. E. F. N. (1999). Análise de modelo afetivo para intenção de rotatividade [Resumo], I Congresso Norte-Nordeste de Psicologia, Anais, Salvador.

Souza, M. A., \& Ribas Júnior, R. C. (2013). Anomia organizacional: Discussão conceitual e desenvolvimento de escala. Psicologia Argumento (PUCPR. Online), 31(75), 677686.

Spector, P. E. (2002). Psicologia nas organizações. São Paulo: Saraiva.

Tsahuridu, E. (2006). Anomie and ethics at work. Journal of Business Ethics, 69(2), 163174.

Tsahuridu, E. (2011). An Exploration of Factors Affecting Work Anomia. Journal of Business Ethics 99(2), 29-305. doi: 10.1007/s10551-010-0655-2

Van der Heijden, B. I. J. M.; Peeters, M. C. W.; Le Blanc, P. M. \& Van Breukelen, J. W. M. (2018). Job characteristics and experience as predictors of occupational turnover intention and occupational turnover in the European nursing sector. Journal of Vocational Behavior, 108, 108-120. doi: 10.1016/j.jvb.2018.06.008

Wiener, Y. (1982). Commitment in organizations: a normative view. The Academy of Management Review, 7(3), 418-428.

Yao, T., Qiu, Q., \& Wei, Y. (2018). Retaining hotel employees as internal customers: Effect of organizational commitment on attitudinal and behavioral loyalty of 
employees. International Journal of Hospitality Management, 76, 1-8. doi: 10.1016/j. ijhm.2018.03.018

Zhao, R. \& Cao, L. (2010). Social Change and Anomie: A Cross-national Study. Social Forces, 88(3), 1209-1229. doi: 10.1353/sof.0.0312

Recebido: 25 de agosto, 2017

Aceitado: 19 de julho, 2018 